Provided for non-commercial research and education use. Not for reproduction, distribution or commercial use.

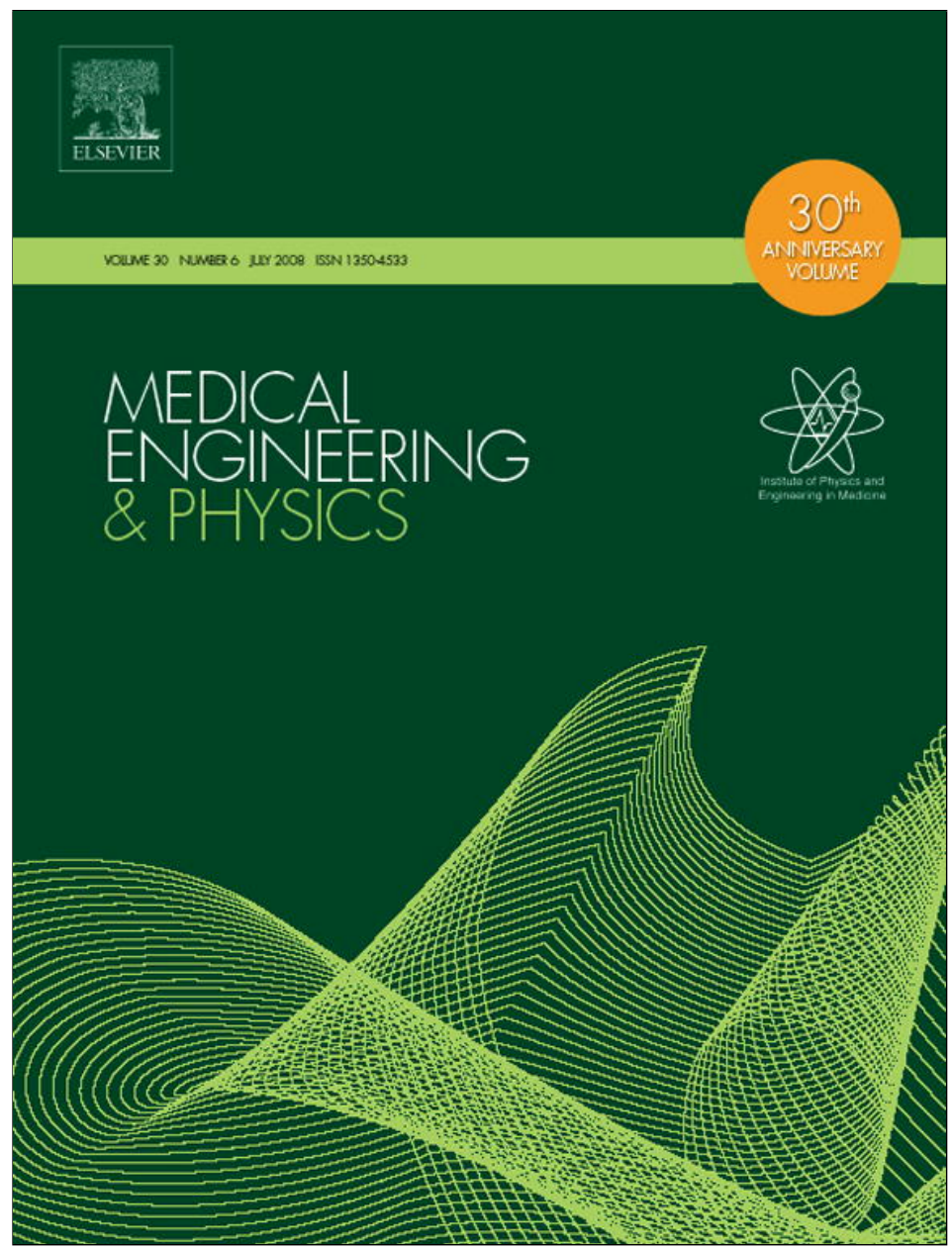

This article appeared in a journal published by Elsevier. The attached copy is furnished to the author for internal non-commercial research and education use, including for instruction at the authors institution and sharing with colleagues.

Other uses, including reproduction and distribution, or selling or licensing copies, or posting to personal, institutional or third party websites are prohibited.

In most cases authors are permitted to post their version of the article (e.g. in Word or Tex form) to their personal website or institutional repository. Authors requiring further information regarding Elsevier's archiving and manuscript policies are encouraged to visit:

http://www.elsevier.com/copyright 


\title{
An algorithm to allow humerus translation in the indeterminate problem of shoulder abduction
}

\author{
Alexandre Terrier ${ }^{\mathrm{a}, *},{\text { Arne } \text { Vogel }^{\mathrm{a}} \text {, Massimiliano Capezzali }}^{\mathrm{a}}$, Alain Farron ${ }^{\mathrm{b}}$ \\ a Laboratory of Biomechanical Orthopedics Ecole Polytechnique Fédérale de Lausanne, Lausanne, Switzerland \\ ${ }^{\mathrm{b}}$ Department of Orthopaedics and Traumatology Centre Hospitalier Universitaire Vaudois and \\ University of Lausanne, Lausanne, Switzerland \\ Received 23 November 2006; received in revised form 18 June 2007; accepted 24 July 2007
}

\begin{abstract}
The shoulder is one of the most complex joints of the human body, mainly because of its large range of motion, but also because of its active muscular stabilisation. Actually, the numerous stabilizing muscles and degrees of freedom yield indeterminate biomechanical models. To solve this indeterminate, most models use reverse dynamics with a simplified ball-socket joint, preventing therefore the natural humerus translation. In this paper, an algorithm was specifically developed to solve the indeterminate problem by a feedback control of muscle activation, allowing the natural humerus translation. Abduction was considered in the scapular plane, accounting for the three deltoid parts and the rotator cuff muscles. The major aim of this study was to validate the numerical algorithm, which was here restricted to two-dimensions in order to compare the numerical solution to a known algebraic one. This comparison gave a relative error below $0.1 \%$. The joint reaction force was comparable to other models and the humerus translation was in agreement with in vivo or in vitro studies.
\end{abstract}

(C) 2007 IPEM. Published by Elsevier Ltd. All rights reserved.

Keywords: Biomechanics; Finite element method; Shoulder

\section{Introduction}

Even when the biomechanical analysis of the shoulder is focused on the glenohumeral joint, and when the motion is restricted to abduction in the scapular plane, the estimation of the joint reaction force remains challenging. The difficulty is mainly caused by the indeterminate of the muscle forces, e.g. [1], but also by the limited intrinsic stability of the joint. Although the glenohumeral joint is often assumed as a ball-socket joint, natural translations of the humeral head are observed in vitro and in vivo. It is clear that these translations are a key factor in the study of the glenohumeral joint stability, particularly to analyse the biomechanics and survival of artificial replacement of this joint [2].

\footnotetext{
* Corresponding author. Laboratory of Biomechanical Orthopedics, STI-IBME-LBO, Bâtiment AI, Station 15, Ecole Polytechnique Fédérale de Lausanne, 1015 Lausanne, Switzerland.

E-mail address: alexandre.terrier@epfl.ch (A. Terrier).
}

Until now, most biomechanical models of the shoulder, e.g. [1,3-5], assume that the glenohumeral joint is a ball-socket joint. By this simplification, the amplitude and location of the joint contact force can be easily estimated, but the natural translations are forbidden and the resulting glenoid stress might be underestimated. The ball-socket assumption is based on the observation that, on average, the glenoid and humeral articular surfaces are almost spherical and congruent, e.g. [6]. It is also justified by the limited translation ranges observed in cadaver [7] or in vivo studies [8-10], although larger translation ranges were also observed [11]. Most studies report an initial superior translation of 2-3 mm from 0 to 30 degrees of abduction, followed by a more stable phase up to full abduction. From roentgenogram measurements on healthy volunteers, Poppen and Walker [9] concluded that the humeral head is fairly stable above 60 degrees of abduction, with only 1-2 mm of upward or downward translation every 30 degrees. With a similar method, Deutsh et al. [8] observed no significant translation of the humerus from 0 to 120 degrees of abduction. Using open 
MRI on healthy volunteers, Graichen et al. [10] observed an average downward translation of $1 \mathrm{~mm}$ after the initial upward translation, and a centering of the joint between 90 and 120 degrees of abduction. In a cadaver study [7], the upward and downward translation of the humerus during shoulder abduction was clearly observed, in a range of about $2 \mathrm{~mm}$.

Although these studies seem to validate the ball-socket assumption, the humerus translation remains a key point in the stability analysis of the glenohumeral joint. This is certainly even more crucial for the analysis of less conforming articular prosthesis, where eccentric loading can induce high glenoid stress. Besides, the ball-socket assumption prevents any estimate of the contact area and contact pressure. There is therefore a lack of a biomechanical model that can predict this natural translation.

The goal of the present work was thus to develop a feedback algorithm to control muscle activation during shoulder abduction, in order to allow and predict the natural humerus translation. To validate the numerical algorithm by a comparison with an algebraic solution, the present model was restricted to 2D. The humerus translation was predicted during active and continuous abduction in the scapular plane, for a healthy shoulder and for a pathologic shoulder with deficient rotator cuff muscles. The effect of an additional load in the hand was also investigated.

\section{Methods}

The present numerical model is an extension of an algebraic model (Appendix A). The algebraic model assumes a ball-socket glenohumeral joint, which is first reproduced by the numerical model and then extended to account for the glenohumeral contact allowing natural humerus translation. Shoulder abduction was achieved in the plane of the scapula, from $0^{\circ}$ to $180^{\circ}$ of elevation. Six muscles were considered: middle deltoid (MD), anterior deltoid (AD), posterior deltoid (PD), supraspinatus (SS), subscapularis (SC) and infraspinatus combined with teres minor (IS). Muscle origin and insertion points were estimated from dissection of a cadaver shoulder [12] and projected on the scapular plane (defined by the angulus inferior, the trigonum scapulae and the most dorsal point of the acromioclavicular joint). Muscles were considered as cables with no bending stiffness, but high longitudinal stiffness. The contacts between the MD and the greater tuberosity, and between the SS and the articular surface of the humerus were accounted for. These two bone contours were represented by two rigid circles, centred on the humeral head. Their radius $R^{\mathrm{MD}}$ and $R^{\mathrm{SS}}$ were chosen to best fit the middle line of the MD and the SS when they are in contact with the humeral head.

Without loss of generality, the muscle indeterminate was described by 5 ratios relating the amplitude of each muscle force $F^{K}$ with the one of the MD, which was chosen as the reference muscle because of its major role in shoulder abduction:

$$
\begin{aligned}
& r^{\mathrm{MD}}=\frac{F^{\mathrm{MD}}}{F^{\mathrm{MD}}} \equiv 1, r^{\mathrm{AD}}=\frac{F^{\mathrm{AD}}}{F^{\mathrm{MD}}}, r^{\mathrm{PD}}=\frac{F^{\mathrm{PD}}}{F^{\mathrm{MD}}}, \\
& r^{\mathrm{SS}}=\frac{F^{\mathrm{SS}}}{F^{\mathrm{MD}}}, r^{\mathrm{IS}}=\frac{F^{\mathrm{IS}}}{F^{\mathrm{MD}}}, r^{\mathrm{SC}}=\frac{F^{\mathrm{SC}}}{F^{\mathrm{MD}}}
\end{aligned}
$$

In the algebraic model, these ratios reduced the number of unknowns, making the system solvable. In the numerical model, these ratios were constrained by a user-defined element (UEL). This UEL synchronised the muscle forces through a feedback mechanism controlled by the length of the MD, which also controlled the abduction of the arm. To elevate the arm, the length of the MD was shortened, generating a reaction force within the $\mathrm{MD}$, which was continuously reintroduced within the other 5 muscles, according to predefined muscle ratios. By this way, the equilibrium equations, the contact constraint and the predefined ratios were simultaneously satisfied. The UEL contains one pair of nodes for each of the 6 muscles. The first two nodes are used as force captor within the MD, while the other 5 pairs of node are used to set the force amplitude and direction to the other 5 muscles according to the muscle ratios (Fig. 1). The contribution of this element to the Jacobian operator matrix is

$K^{N M}=-\frac{\mathrm{d} F^{N}}{\mathrm{~d} u^{M}}$

where $F^{N}$ is the contribution of this element to the residual vector force, and $u^{M}$ is the nodal displacement. $N$ and $M$ vary

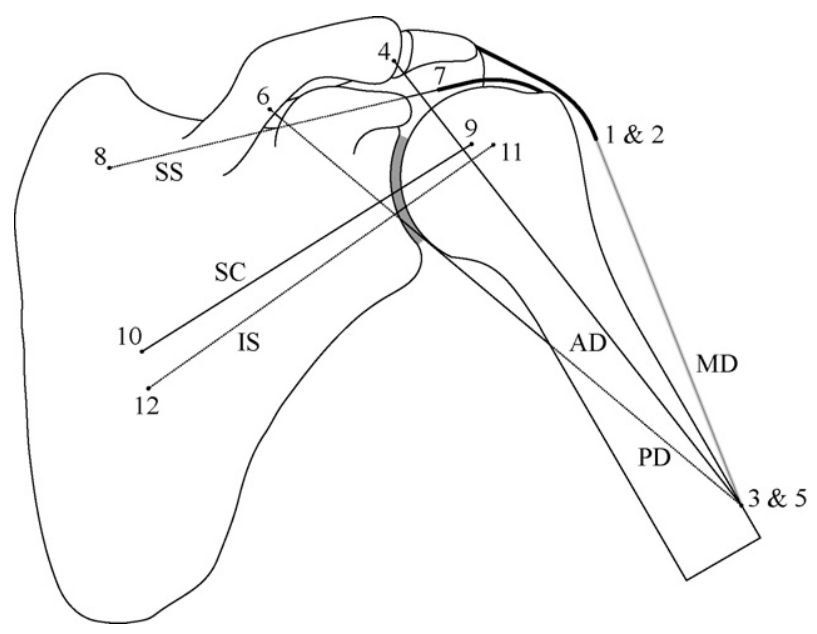

Fig. 1. Numerical shoulder model showing the muscles and their associated node numbers within the user-defined element (UEL). The heavy black curves represent the deformable parts of the MD and the SS, which may be in contact with the humerus and wrap on it. The grey heavy line represents the contractile part of MD. The other lines represent the direction of the muscles forces, whose amplitude is controlled by the UEL. Nodes 1 and 2 are located between the deformable part and the contractile part of the MD; node 1 is connected to the contractile part and node 2 to the deformable part; they are linked to each other by the force captor. Node 7 is linked to the deformable part of the SS. The other nodes are directly linked to humerus insertions or scapula origins. 
from 1 to 24 (6 node pairs times 2 dimensions). Each $F^{N}$ is clearly a function of $u^{1}$ to $u^{4}$, the displacements of the first 2 nodes (force captor) in the two directions. They are related to the elongation of the force captor, and thus to its force amplitude. Each $F^{N}$ is also a function of 4 other displacements $u^{i}$, the 2D displacements of the node pair defining this force direction. An exact algebraic expression for all vector elements $F^{N}$ and matrix elements $K^{N M}$ was obtained and implemented in the UEL. The UEL was introduced as a subroutine of the FE solver Abaqus (Abaqus, Inc.). Since this subroutine is called at each iteration of the nonlinear solving process (Newton-Raphson), the ratios condition is fulfilled at each equilibrium increment of the implicit solving algorithm.

The synchronizing UEL was not connected the same way to each muscle. The MD had three functions: wrapping around the humeral head, active shortening, and force captor. These three features were assumed by three different parts. First, the wrapping part (heavy black curve in Fig. 1), starting from the muscle origin on the acromion and ending below the greater tuberosity, was a passive cable-like structure made of truss elements (no bending stiffness). The role of this part was to resist tension and transmit the contact force to the humeral head. Then, the active shortening part was achieved by a 2 point connector, whose length was controlled as a boundary condition. These two parts were joined together by the third one, the force captor, composed of the first 2 nodes of the UEL. The SS was composed of two parts: a wrapping part and force part. The wrapping part was made of passive truss elements (as for the MD) and the active force part was composed of a UEL node pair (nodes 7 and 8). The 4 remaining muscles had only an active force part and were defined by UEL node pairs (AD: 3 and 4; PD: 5 and 6; SC: 9 and 10; IS: 11 and 12).

As initially proposed by Poppen and Walker [1], the muscle ratios were estimated from the product between physiological cross-section area (PCSA) and electromyographic data (EMG). Since muscular force is proportional to EMG and PCSA, e.g. [13], the ratios are also proportional to relative PCSA and EMG. EMG and PSCA were roughly estimated from literature [13-15] and normalized to the MD (Table 1). Besides, since EMG remains nearly proportional during scapular abduction [13], the ratios were assumed constant as a first approximation.

For the glenohumeral joint, two cases were considered: a ball-socket joint (BS) and an articular contact (AC). The first case corresponded to the algebraic model, prohibiting the natural humerus translation, while the second case allowed this

Table 1

The muscle ratios were roughly estimated by the product between relative PCSA and EMG, which were obtained from literature and normalized to the $\mathrm{MD}$

\begin{tabular}{lllllll}
\hline & MD & AD & PD & SS & SC & IS \\
\hline PCSA & 1.0 & 1.0 & 1.0 & 0.5 & 1.5 & 1.5 \\
EMG & 1.0 & 0.8 & 0.2 & 1.0 & 0.3 & 0.3 \\
Ratios & 1.0 & 0.8 & 0.2 & 0.5 & 0.5 & 0.5 \\
\hline
\end{tabular}

translation. Note that the BS case corresponded exactly to the algebraic model, and should therefore provide the same solution. In the AC case, the real contact between the two articular surfaces was considered. The humeral articular surface was assumed rigid and circular, with a radius of $24 \mathrm{~mm}$. For the glenoid side, a deformable layer of cartilage was used. The shape of this cartilage layer was built from two circular contours. A first circle was fitted to the glenoid bone, resulting in a radius of $30 \mathrm{~mm}$. The second circle, representing the articular side, was chosen to have a radius $2 \mathrm{~mm}$ greater than radius of the humeral head [6,7], and was positioned to set the cartilage thickness in the middle of the glenoid at $2 \mathrm{~mm}$ [6]. These two circles were then limited in the superior and inferior part by two radius lines, forming an angle of 66 degrees [16]. The deformation law of the cartilage layer was based on a Neo-Hookean potential $w=1.8\left(I_{1}-3\right)$, where $I_{1}$ is the first invariant of the right Cauchy-Green stress tensor [17]. The cartilage was filled with 4-nodes bilinear elements having an average size of $0.7 \mathrm{~mm}$. The contact between the glenoid and the humerus and the contacts between the muscles and the humerus were frictionless. They were solved by the default Lagrange multiplier method of the solver. All convergence criteria were left to the solver default values.

As in the algebraic model, the scapula rotation was characterized by a constant scapulohumeral rhythm of 2:1 (for 150 degrees of arm elevation, 50 are completed in the scapulothoracic joint and 100 in the glenohumeral joint). In the algebraic model, this constraint provided a directed relationship between the glenohumeral and scapulothoracic angles (Eq. (2) of the Appendix A). In the numerical model, this constraint was satisfied by setting the rotation of the scapula as a boundary condition, while the MD shortening induced elevation. In the initial configuration, the scapula was placed such that the glenoid axis was horizontal and the diaphyseal axis of the humerus was vertical, corresponding by definition to zero scapulothoracic and scapulohumeral angles. The arm mass was set to $3.75 \mathrm{~kg}$ ( $5 \%$ of a $75 \mathrm{~kg}$ person). All biomechanical parameters of the numerical model were equivalent to the algebraic model (Table A1 of the Appendix A).

Several quantities were calculated: the glenohumeral force, the glenohumeral contact point position, and the humerus translation. In the BS case, as in the algebraic (and other ball-socket models), the contact point location was estimated from the direction of the reaction force that constrains the humerus on its centre of rotation. In the AC case, the real contact position between the two articular surfaces was calculated. This point corresponds to the application point of the resultant contact force producing no moment of force. The contact point position was represented by its angular position above the glenoid centre line. In the AC case, the humerus translation was measured by the inferior-superior position of the humeral head centre relative to the glenoid cavity centre. Zero translation corresponded to a perfect centring of the joint. The numerical accuracy of the model was determined by comparison of the BS case with the exact algebraic solution. The deficiency of the rotator cuff was investigated by 


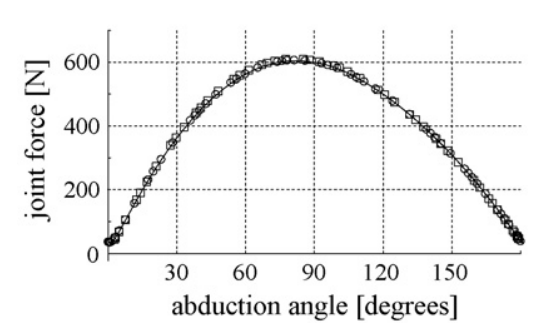

- BS algebraic solution

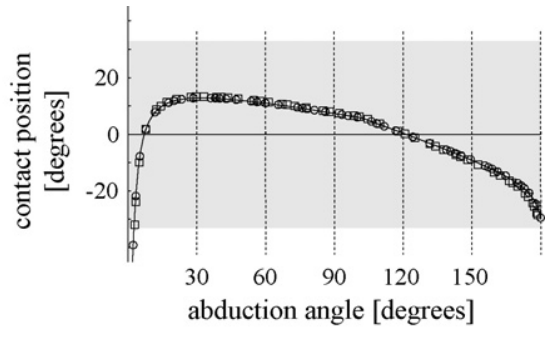

- AC numerical solution

Fig. 2. Joint reaction force (left) and contact position (right) for the BS algebraic solution, the BS numerical solution and the AC numerical solution. On the right, the gray zone corresponds approximately to subluxation limits.
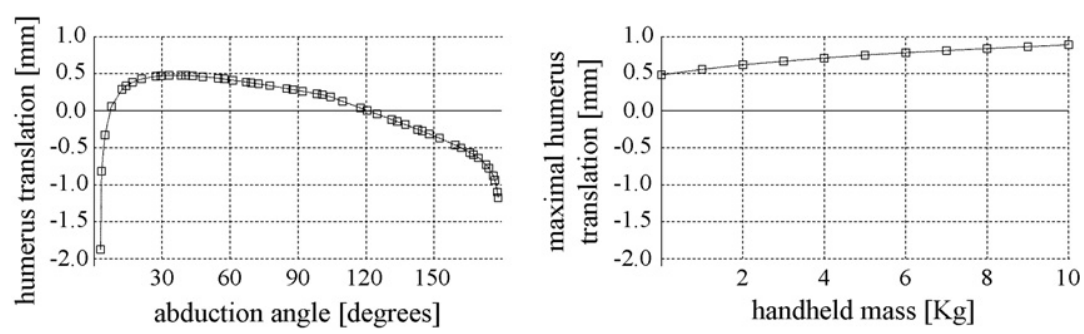

Fig. 3. Left: inferior-superior humerus translation relative to the glenoid vs. abduction angle for an unloaded arm. Zero translation corresponds to exact centring of the humeral head in the joint. Right: maximal humerus translation vs. additional handheld mass.

setting the corresponding muscles ratios to zero. The effect of an increasing handheld loading was evaluated up to $10 \mathrm{~kg}$. The hand position was estimated at $640 \mathrm{~mm}$ from the humeral head centre, on the humeral axis [1].

\section{Results}

The glenohumeral force and the contact point location were very close in the algebraic, BS and AC cases (Fig. 2). The accuracy of the numerical model was very high: the difference between the numerical and the exact algebraic solution was only $0.1 \%$. The global convergence of the model was also very good: only 50 non-linear iterations of the implicit solver were required for the entire abduction range.

For an unloaded arm, the humerus translated upwards by $2.5 \mathrm{~mm}$, from 0 to 30 degrees of abduction. At 30 degrees of abduction, the centre of the humeral head was however only $0.5 \mathrm{~mm}$ above the centre of the glenoid cavity (Fig. 3). Above 30 degrees, but mainly from 60 degrees, the humeral head moved downwards. It was again centred at 120 degrees of abduction and was about 0.5 below the joint centre at 160 degrees of abduction.

When the rotator cuff muscles were deactivated (massive tendon tear), the remaining deltoid activation induced an instantaneous upward migration of the humeral head, instead of a normal abduction. As a consequence, the contact point also moved immediately to the glenoid superior edge. The humerus translation reached $5 \mathrm{~mm}$, moving the humeral head centre $3 \mathrm{~mm}$ above the joint centre. In fact, the humerus translation was stopped when the MD contact circle $\left(R^{\mathrm{MD}}=30 \mathrm{~mm}\right)$ eached the origin point of the MD $(33 \mathrm{~mm}$ above centre). This limit corresponds to the contact between the SS and the acromion arch.

When an additional mass was placed in the hand, and increased up to $10 \mathrm{~kg}$, the maximal translation of the humerus above the joint centre continuously increased from 0.5 to $0.9 \mathrm{~mm}$ (Fig. 3). The position of the contact point was almost not influenced by the handheld load. With $10 \mathrm{~kg}$ in the hand, the glenohumeral and MD forces reached, respectively, 4000 and $1200 \mathrm{~N}$.

\section{Discussion}

The present work was a preliminary step towards a 3D model of the shoulder. In this paper, the algebraic model was extended to allow and predict the natural translation of the humerus during shoulder abduction. The comparison with the algebraic solution confirmed the validity of the numerical algorithm, and thus the feasibility of the method for the generalization to 3D. Predicted humerus translations confirmed the function of the muscles of the rotator cuff for the joint stability, even for extreme loading.

The glenohumeral contact force and the contact point position were very similar for the algebraic, BS and AC cases. The comparison between the algebraic solution and BS case demonstrated that the numerical model, and particularly the user-defined element subroutine, provides a very accurate solution of the biomechanical model. The comparison between the $\mathrm{BS}$ and $\mathrm{AC}$ cases confirmed that the ball-socket approximation is valid for a force analysis of the glenohumeral joint. The important superior translation occurring in the first 30 degrees of abduction corresponded to the 
initial centring of the humeral head in the glenoid fossa. After this initial centring of the joint, the upward and downward translation was caused by the orientation of the muscle forces resultant, which changed during abduction. The resultant orientation was indeed above the glenoid axis up to 120 degrees of abduction, and below thereafter. The predicted translation range could certainly be associated to the joint conformity and congruency, as reported by, e.g. [7]. These two geometric parameters were however not investigated in the present study, since only average values were tested [6].

The glenohumeral force and the location of the contact position were consistent with other studies, e.g. [1,5], despite some discrepancy in the maximal glenohumeral force. The predicted humerus translation was also in good agreement with in vivo and in vitro studies. A radiographic study on healthy volunteers reported an upward motion of about $3 \mathrm{~mm}$ from 0 to 30 degrees and a stabilization thereafter (only $1 \mathrm{~mm}$ upward or downward every 30 degrees) [9]. In another in vivo study, open MRI was used to measure the humerus translation on healthy subjects [10]. In average, the centre of the humerus was $1 \mathrm{~mm}$ above the centre of the glenoid at 60 degrees and was centred between 90 and 120 degrees. In a cadaver study, using also constant muscle force ratios, but no scapular motion, the average inferior-superior translation was more than $3 \mathrm{~mm}$ from 20 to 30 degrees, and continuously increased from 30 to 90 degrees, up to $9 \mathrm{~mm}$ [11], which seems contradictory with other studies. More recently, another cadaver study using predetermined muscle ratios and accounting for scapular motion measured humerus translation every 10 degrees of abduction [7]. This study reported an initial upward translation of $1 \mathrm{~mm}$ from 10 to 30 degrees, followed by a downward translation of $1.5 \mathrm{~mm}$ from 60 to 150 degrees.

The restriction to $2 \mathrm{D}$ remains the main limitation of the present model, since it prevents the estimation of the real contact area and therefore the contact pressure within the joint. This will be the next step of this modelling work of the shoulder. The main issue of this three-dimensional extension will be to stabilize the muscles that wrap around the humeral head. In 2D, the stability of the cable-like MD and SS muscles around infinite cylinder was obvious and natural, which will not be the case in $3 \mathrm{D}$, around the spherical shape of the humeral head. Besides, the wrapping of SC and IS muscles will have to be added. The stability of the humerus will also be more difficult since 3 more degrees of freedom will be released: the anterior-posterior translation and two rotations. Therefore, the increase of complexity from the current 2D model towards its 3D extension will be made progressively, allowing for a continuous check from the present $2 \mathrm{D}$ reference.

To our knowledge, only a few cadaver or in vivo studies measured the natural translation of the humerus during abduction, and most computer or theoretical models prevent this translation by the use of the simplifying ball-socket assumption. The present paper proposes an original numerical algorithm to analyze the biomechanics of the shoulder, and to predict natural humerus translation in agreement with in vivo and in vitro studies. This model also confirms the crucial role of the rotator cuff muscles to stabilize the glenohumeral joint, even for extreme loading. Finally, the present work is a preliminary feasibility study for a more complex 3D model, which should be a valuable tool to relate the survival of artificial shoulder replacements with critical humerus translations.

\section{Acknowledgments}

This study was partly supported by Tornier S.A.S. (Montbonnot, France).

\section{Appendix A}

For this two-dimensional algebraic model of shoulder abduction, the glenohumeral joint is assumed to be a ballsocket joint. The muscle indeterminate is represented by 5 ratios, which are a priori unknown (Fig. A1):

$$
\begin{aligned}
& r^{\mathrm{MD}}=\frac{F^{\mathrm{MD}}}{F^{\mathrm{MD}}} \equiv 1, r^{\mathrm{AD}}=\frac{F^{\mathrm{AD}}}{F^{\mathrm{MD}}}, r^{\mathrm{PD}}=\frac{F^{\mathrm{PD}}}{F^{\mathrm{MD}}}, \\
& r^{\mathrm{SS}}=\frac{F^{\mathrm{SS}}}{F^{\mathrm{MD}}}, r^{\mathrm{IS}}=\frac{F^{\mathrm{IS}}}{F^{\mathrm{MD}}}, r^{\mathrm{SC}}=\frac{F^{\mathrm{SC}}}{F^{\mathrm{MD}}}
\end{aligned}
$$

relating the force amplitude of each muscle to the one of the MD. Muscles are represented by cables and only the MD and the SS can wrap on the humeral head. For these two contacts, the bony side is represented by two rigid circles, with radius $R^{\mathrm{MD}}$ and $R^{\mathrm{SS}}$. As in rope-pulley problems, continuous contact forces are replaced by a single force. The abduction angle $\alpha$ is decomposed into glenohumeral $\alpha_{g h}$ and scapulothoracic $\alpha_{\text {st }}$ angle according to the scapulohumeral rhythm:

$$
\alpha=\alpha_{\mathrm{gh}}+\alpha_{\mathrm{st}}, \quad \frac{\alpha_{\mathrm{gh}}}{\alpha_{\mathrm{st}}}=2
$$

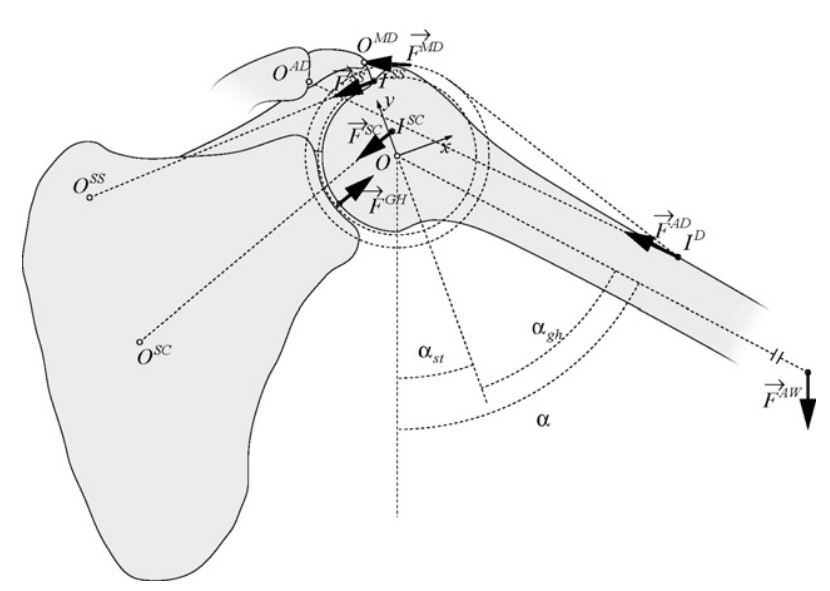

Fig. A1. The algebraic shoulder model with the local reference frame $O x y$, the abduction angles $\left(\alpha, \alpha_{\mathrm{st}}, \alpha_{\mathrm{gh}}\right)$, the origins $O^{K}$, insertions $I^{K}$, muscle forces $\vec{F}^{K}$, glenoid reaction $\vec{F}^{\mathrm{GH}}$, and arm weight $\vec{F}^{\mathrm{AW}}$. The two contact circles are also represented. 
At equilibrium,

$\sum_{K} \vec{F}^{K}=\overrightarrow{0}$

$\sum_{K} \vec{X}^{K} \times \vec{F}^{K}=\overrightarrow{0}$

The 8 forces $\vec{F}^{K}$ (6 muscles, glenoid reaction, and arm weight) are described in a local coordinate system $O x y$ fixed to the scapula (origin $O$ on the humeral head centre and $y$ parallel to the glenoid):

$\vec{F}^{K}=\left(\begin{array}{c}F_{x}^{K} \\ F_{y}^{K}\end{array}\right)=F^{K}\left(\begin{array}{c}\cos \alpha_{F^{K}} \\ \sin \alpha_{F^{K}}\end{array}\right)$

$\vec{X}^{K}=\left(\begin{array}{c}X_{x}^{K} \\ X_{y}^{K}\end{array}\right)=X^{K}\left(\begin{array}{c}\cos \alpha_{X^{K}} \\ \sin \alpha_{X^{K}}\end{array}\right)$

For the muscles, the amplitude $F^{K}$ is unknown, but $X^{K}, \alpha_{X^{K}}$ and $\alpha_{F^{K}}$ are determined geometrically. When the muscle is not in contact with the humeral head, $\vec{X}^{K}=\vec{I}^{K}$, where

$\vec{I}^{K}=\left(\begin{array}{c}I_{x}^{K} \\ I_{y}^{K}\end{array}\right)=\left(\begin{array}{cc}I_{x 0}^{K} & \cos \left(\alpha_{g h}\right)-I_{y 0}^{K} \sin \left(\alpha_{g h}\right) \\ I_{y 0}^{K} & \cos \left(\alpha_{g h}\right)+I_{x 0}^{K} \sin \left(\alpha_{g h}\right)\end{array}\right)$

is the position of each muscle insertion, and $\left(I_{x 0}^{K}, I_{y 0}^{K}\right)$ are $\vec{I}^{K}$ coordinates in the initial configuration $(\alpha=0)$. Angles $\alpha_{X^{K}}$ and $\alpha_{F^{K}}$ are given by

$\alpha_{X^{K}}=\left\{\begin{array}{cc}\sin ^{-1}\left(\frac{X_{y}^{K}}{X^{K}}\right), & X_{x}^{K} \geq 0 \\ \pi-\sin ^{-1}\left(\frac{X_{y}^{K}}{X^{K}}\right), & X_{x}^{K}<0\end{array}\right.$

$\alpha_{F^{K}}=\pi-\sin ^{-1}\left(\frac{O_{y}^{K}-X_{y}^{K}}{\sqrt{\left(O_{x}^{K}-X_{x}^{K}\right)^{2}+\left(O_{y}^{K}-X_{y}^{K}\right)^{2}}}\right)$

where $\left(O_{x}^{K}, O_{x}^{K}\right)$ are the muscles origin coordinates. When the MD or the SS are in contact expressions of $X^{K}, \alpha_{X^{K}}$ and $\alpha_{F^{K}}$ are different: $X^{K}=R^{K}$,

$\alpha_{X^{K}}=\sin ^{-1}\left(\frac{X^{K}}{O^{K}}\right)-\sin ^{-1}\left(\frac{O_{x}^{K}}{O^{K}}\right)$

$\alpha_{F^{K}}=\alpha_{X^{K}}+\frac{\pi}{2}$

with $O^{K}=\sqrt{\left(O_{x}^{K}\right)^{2}+\left(O_{y}^{K}\right)^{2}}$. The contact state (contact/no contact) of the MD and the SS, respectively, switches when

$$
\begin{aligned}
\alpha_{\mathrm{gh}}>\alpha_{\mathrm{gh}}^{\mathrm{MD}}= & \sin ^{-1}\left(\frac{R^{\mathrm{MD}}}{O^{\mathrm{MD}}}\right)-\sin ^{-1}\left(\frac{O_{x}^{\mathrm{MD}}}{O^{\mathrm{MD}}}\right) \\
& +\sin ^{-1}\left(\frac{R^{\mathrm{MD}}}{I^{\mathrm{MD}}}\right)-\sin ^{-1}\left(\frac{I_{X}^{\mathrm{MD}}}{I^{\mathrm{MD}}}\right)
\end{aligned}
$$

\begin{tabular}{|c|c|c|}
\hline Parameter & Symbol & Value \\
\hline \multicolumn{3}{|l|}{ Muscle origins [mm] } \\
\hline Middle deltoid & $\left(O_{x}^{\mathrm{MD}}, O_{y}^{\mathrm{MD}}\right)$ & $(2,33)$ \\
\hline Anterior deltoid & $\left(O_{x}^{\mathrm{AD}}, O_{y}^{\mathrm{AD}}\right)$ & $(-15,29)$ \\
\hline Posterior deltoid & $\left(O_{x}^{\mathrm{PD}}, O_{y}^{\mathrm{PD}}\right)$ & $(-55,23)$ \\
\hline Supraspinatus & $\left(O_{x}^{\mathrm{SS}}, O_{y}^{\mathrm{SS}}\right)$ & $(-81,25)$ \\
\hline Subscapularis & $\left(O_{x}^{\mathrm{SC}}, O_{y}^{\mathrm{SC}}\right)$ & $(-99,-23)$ \\
\hline Infraspinatus & $\left(O_{x}^{\stackrel{I}{I S}}, O_{y}^{I S}\right)$ & $(-97,-22)$ \\
\hline \multicolumn{3}{|c|}{ Muscle insertions [mm] } \\
\hline Middle deltoid & $\left(I_{x 0}^{\mathrm{MD}}, I_{y 0}^{\mathrm{MD}}\right)$ & $(20,-90)$ \\
\hline Anterior deltoid & $\left(I_{x 0}^{\mathrm{AD}}, I_{y 0}^{\mathrm{AD}}\right)$ & $(20,-90)$ \\
\hline Posterior deltoid & $\left(I_{y 0}^{\mathrm{PD}}, I_{y 0}^{\mathrm{PD}}\right)$ & $(20,-90)$ \\
\hline Supraspinatus & $\left(I_{r 0}^{S S}, I_{r 0}^{S S S}\right)$ & $(23,13)$ \\
\hline Subscapularis & $\left(I_{v 0}^{\mathrm{SC}}, I_{r 0}^{\mathrm{SC}}\right)$ & $(0,10)$ \\
\hline Infraspinatus & $\left(I_{x 0}^{\mathrm{IS}}, I_{y 0}^{\mathrm{IS}}\right)$ & $(5,9)$ \\
\hline \multicolumn{3}{|l|}{ Muscle ratios } \\
\hline Middle deltoid & $r^{\mathrm{MD}}$ & 1.0 \\
\hline Anterior deltoid & $r^{\mathrm{AD}}$ & 0.8 \\
\hline Posterior deltoid & $r^{\mathrm{PD}}$ & 0.2 \\
\hline Supraspinatus & $r^{\mathrm{SS}}$ & 0.5 \\
\hline Subscapularis & $r^{\mathrm{SC}}$ & 0.5 \\
\hline Infraspinatus & $r^{\mathrm{IS}}$ & 0.5 \\
\hline \multicolumn{3}{|l|}{ Contact radii $[\mathrm{mm}]$} \\
\hline Middle deltoid & $R^{\mathrm{MD}}$ & 30 \\
\hline Supraspinatus & $R^{\mathrm{SS}}$ & 26 \\
\hline \multicolumn{3}{|c|}{ Arm gravity centre $[\mathrm{mm}]$} \\
\hline$X^{\mathrm{A}}$ & 320 & \\
\hline \multicolumn{3}{|l|}{ Arm mass [kg] } \\
\hline$M^{\mathrm{A}}$ & 3.75 & \\
\hline \multicolumn{3}{|c|}{ Scapulohumeral rhythm } \\
\hline & 2 & \\
\hline \multicolumn{3}{|c|}{ Gravity constant $\left[\mathrm{m} / \mathrm{s}^{2}\right]$} \\
\hline$g$ & 10 & \\
\hline
\end{tabular}

Table A1

$\alpha_{\mathrm{gh}}>\alpha_{\mathrm{gh}}^{\mathrm{SS}_{\mathrm{c}}}=\sin ^{-1}\left(\frac{R^{\mathrm{SS}}}{O^{\mathrm{SS}}}\right)-\sin ^{-1}\left(\frac{O_{y}^{\mathrm{SS}}}{O^{\mathrm{SS}}}\right)$

$$
+\sin ^{-1}\left(\frac{R^{\mathrm{SS}}}{I^{\mathrm{SS}}}\right)-\sin ^{-1}\left(\frac{I_{Y}^{\mathrm{SS}}}{I^{\mathrm{SS}}}\right)
$$

with $I^{K}=\sqrt{\left(I_{X}^{K}\right)^{2}+\left(I_{Y}^{K}\right)^{2}}$. The amplitude $F^{\mathrm{AW}}$ of the arm weight is fixed, and its orientation is $\alpha_{F} \mathrm{AW}=3 / 2 \pi-\alpha_{\mathrm{st}}$, according to Eq. (2). The distance $X^{\mathrm{AW}}$ of its application point from the origin is also fixed, and its direction is $\alpha_{X^{\mathrm{AW}}}=$ $3 / 2 \pi+\alpha-\alpha_{\text {st }}$. The amplitude $F^{\mathrm{GH}}$ and direction $\alpha_{F^{\mathrm{GH}}}$ of the glenohumeral reaction are unknown, but the frictionless ball-socket joint gives

$\alpha_{X^{\mathrm{GH}}}=\pi-\alpha_{F^{\mathrm{GH}}}$

Finally, only three unknowns remain: $F^{\mathrm{MD}}, F^{\mathrm{GH}}$ and $\alpha_{F} \mathrm{GH}$. The moment Eq. (4) gives:

$F^{\mathrm{MD}}=F^{\mathrm{AW}} \frac{X^{\mathrm{AW}} \sin (\alpha)}{\sum_{K^{r^{K}} X^{K} \sin \left(\Delta \alpha^{K}\right)}}$ 
with $K=\mathrm{AD}, \mathrm{PD}, \mathrm{SS}, \mathrm{SC}, \mathrm{IS}$. Note that $X^{K}$ and $\Delta \alpha^{K}=\alpha_{F^{K}}-$ $\alpha_{X^{K}}$ depend on $\alpha$ through Eqs. (5)-(13). The force amplitude of all other muscles is determined from the ratios $r^{K}$ Eq. (1), while the amplitude $F^{\mathrm{GH}}$ and orientation $\alpha_{F} \mathrm{GH}$ of the glenoid reaction are obtained from the force Eq. (3).

\section{Conflict of interest}

None.

\section{References}

[1] Poppen NK, Walker PS. Forces at the glenohumeral joint in abduction. Clin Orthop 1978;135:165-70.

[2] Karduna AR, et al. Glenohumeral joint translations before and after total shoulder arthroplasty. A study in cadavera. J Bone Joint Surg Am 1997;79(8):1166-74

[3] Inman VT, Saunders JB, Abbott LC. Observations of the function of the shoulder joint. J Bone Joint Surg Am 1944;26:1-30.

[4] Karlsson D, Peterson B. Towards a model for force predictions in the human shoulder. J Biomech 1992;25(2):189-99.

[5] van der Helm FC. Analysis of the kinematic and dynamic behavior of the shoulder mechanism. J Biomech 1994;27(5):527-50.

[6] Soslowsky LJ, et al. Articular geometry of the glenohumeral joint. Clin Orthop 1992;285:181-90.
[7] Kelkar R, et al. Glenohumeral mechanics: a study of articular geometry, contact, and kinematics. J Shoulder Elbow Surg 2001;10(1):7384.

[8] Deutsch A, et al. Radiologic measurement of superior displacement of the humeral head in the impingement syndrome. J Shoulder Elbow Surg 1996;5(3):186-93.

[9] Poppen NK, Walker PS. Normal and abnormal motion of the shoulder. J Bone Joint Surg Am 1976;58(2):195-201.

[10] Graichen $\mathrm{H}$, et al. Glenohumeral translation during active and passive elevation of the shoulder - a 3D open-MRI study. J Biomech 2000;33(5):609-13.

[11] Wuelker N, et al. Translation of the glenohumeral joint with simulated active elevation. Clin Orthop Relat Res 1994;309:193-200.

[12] Buchler P, et al. A finite element model of the shoulder: application to the comparison of normal and osteoarthritic joints. Clin Biomech 2002;17(9-10):630-9.

[13] Ringelberg JA. EMG and force production of some human shoulder muscles during isometric abduction. J Biomech 1985;18(12):939_ 47.

[14] Johnson GR, et al. Modelling the muscles of the scapula morphometric and coordinate data and functional implications. J Biomech 1996;29(8):1039-51.

[15] Kronberg M, Nemeth G, Brostrom LA. Muscle activity and coordination in the normal shoulder. An electromyographic study. Clin Orthop Relat Res 1990;257:76-85.

[16] McPherson EJ, et al. Anthropometric study of normal glenohumeral relationships. J Shoulder Elbow Surg 1997;6(2):105-12.

[17] Buchler P, Farron A. Benefits of an anatomical reconstruction of the humeral head during shoulder arthroplasty: a finite element analysis. Clin Biomech 2004;19(1):16-23. 